\title{
High Efficiency Phase Contrast Imaging In STEM Using Fast Direct Electron Pixelated Detectors
}

Hao Yang ${ }^{1}$, Lewys Jones ${ }^{1}$, Henning Ryll ${ }^{2}$, Martin Simson $^{3}$, Heike Soltau ${ }^{3}$, Yukihito Kondo ${ }^{4}$, Ryusuke Sagawa $^{4}$, Hiroyuki Banba ${ }^{4}$, Timothy J. Pennycook ${ }^{5}$ and Peter D. Nellist ${ }^{1}$

1. University of Oxford, Department of Materials, Parks Rd, Oxford, UK

2. PNSensor GmbH, Otto-Hahn-Ring 6, 81739 München, Germany

3. PNDetector GmbH, Sckellstraße 3, 81667 München, Germany

4. JEOL Ltd.,3-1-2 Musashino Akishima Tokyo 196-8558 Japan

5. Faculty of Physics, University of Vienna, Vienna, Austria

Phase contrast imaging using electron elastic scattering has been shown to provide the highest efficiency for imaging weak phase objects for a given amount of radiation damage, compared to inelastic electron scattering as well as X-ray and neutron scattering [1]. Phase contrast imaging in scanning transmission electron microscopy (STEM) is attractive because of its flexibility in detector geometries without modifying the main optics of the electron column, and for the ability to simultaneously record analytical signals. Current bright-field STEM detector geometries include annular bright field (ABF) and differential phase contrast (DPC) imaging. As most phase information from weak scattering objects is contained within the bright field (BF) disc of the convergent beam electron diffraction (CBED) pattern, a detector geometry that makes full use of the variations in intensity of the $\mathrm{BF}$ disc is desirable to maximize the image contrast.

We demonstrate high efficiency phase contrast imaging using a fast pixelated detector. By recording the CBED pattern at every probe position forming a 4-dimensional dataset, the phase can be reconstructed using a ptychographic phase reconstruction method based on one developed described by Rodenburg et al. [2,3] Taking the Fourier transform of the 4D dataset with respect to probe position allows interference between the BF discs and specific diffraction angles to be isolated. The interference inside the disc-overlapping region contains phase information, as shown in Figure 1. A phase image can be reconstructed by integrating such disc-overlapping regions for each spatial frequency contained in the final image. Through simulations, we find that for imaging weak phase objects, phase contrast imaging using ptychography with pixelated detectors gives the optimal phase contrast transfer function (PCTF), and generates the best phase contrast and low dose performances compared to existing imaging modes in STEM including BF, ABF and DPC. [4]

Experiments on phase reconstruction using a fast pixelated detectors have been performed, and here we show results of an $\mathrm{Au}$ nanoparticle with five-fold twinning (Figure 2a-d), and gallium nitride GaN

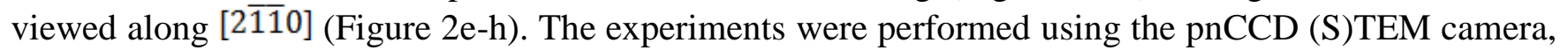
a direct electron pixelated detector from PNDetector, mounted on the JEOL ARM200-CF aberration corrected microscope. The detector has a grid of 264x264 pixels and operates at a speed of 1000 framesper-second (fps). The detector can achieve a speed of up to 20,000 fps through binning/windowing. ADF images can be recorded simultaneously. Using the 4D dataset which records the diffraction patterns that contains the entire $\mathrm{BF}$ and part of DF regions, synthetic BF, ABF and DPC can be obtained along with the ptychographic phase reconstruction. The reconstructed phase of the Au nanoparticle (Figure 2c,d) shows a clear contrast on every atomic column. In comparison, ABF, as a nonlinear imaging mode, shows contrast that decreases towards the edge of the Au particle (Figure 2b). In the case of GaN, the $\mathrm{N}$ columns are hardly visible in the ABF image (Figure 2f), but are clearly resolved in the reconstructed phase (Figure 2g,h).[5] 
[1] Henderson, R. Quarterly Reviews of Biophysics 28, 171-193 (1995).

[2] Rodenburg, J. M. et al. Ultramicroscopy 48, 304-314 (1993).

[3] Pennycook, T. J. et al. Ultramicroscopy. doi:10.1016/j.ultramic.2014.09.013.

[4] Yang, H. et al. Ultramicroscopy. doi:10.1016/j.ultramic.2014.10.013

[5] The authors acknowledge funding from the EPSRC (grant numbers EP/K032518/1 and EP/K040375/1) and the EU Seventh Framework Programme: ESTEEM2.
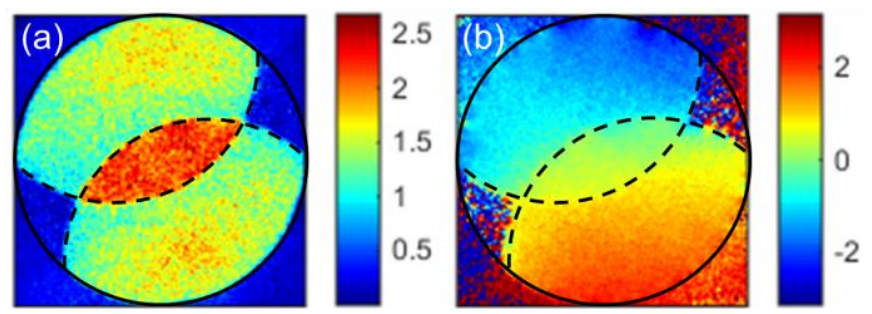

Figure 1. The (a) amplitude and (b) phase of the interference between the central bright field beam (solid circle) and the diffracted beams (dashed circles) in the detector plane at a certain spatial frequency. This experimental result is obtained on an Au nanoparticle, by taking the Fourier transform of a four dimensional dataset which consists of the CBED patterns recorded at every probe position using pixelated detectors. The colour-bar in the amplitude and the phase is in arbitrary unit and in unit of radian, respectively.

(a) ADF

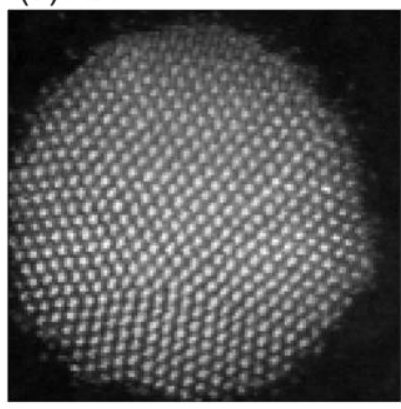

(e) ADF

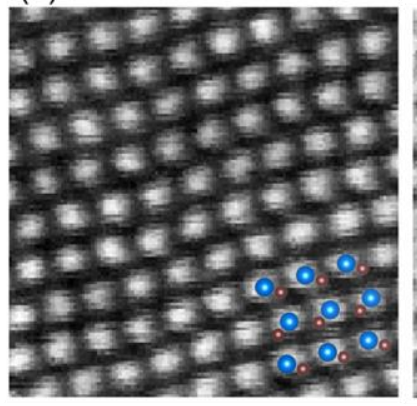

(b) $\mathrm{ABF}$

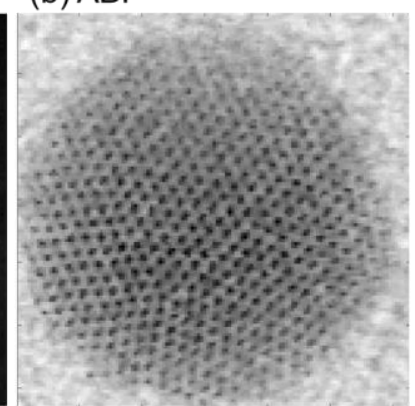

(f) $\mathrm{ABF}$

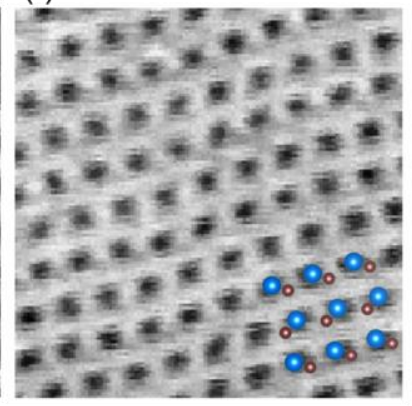

(c) Amplitude

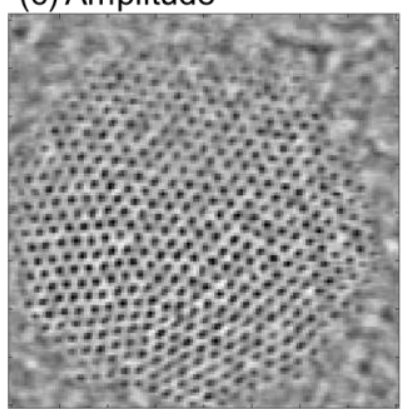

(g) Amplitude

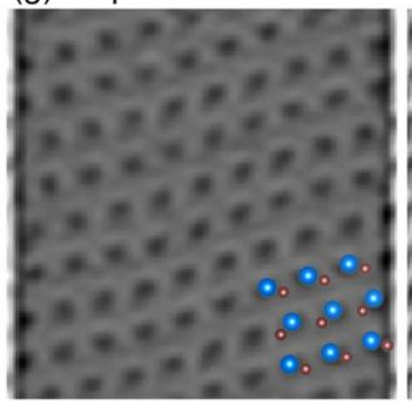

(d) Phase

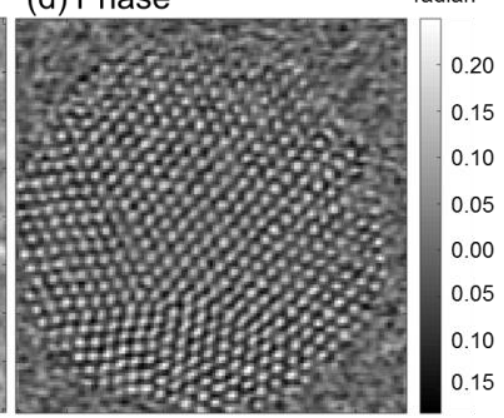

(h) Phase

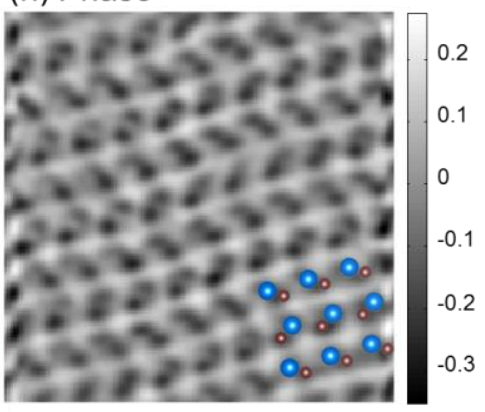

$\bullet \mathrm{Ga} \bullet \mathrm{N}$

Figure 2. Simultaneous atomic resolution ADF, synthetic ABF image, and the reconstructed amplitude and phase of (a-d) an Au nanoparticle and (e-h) GaN bulk lattice viewed along the [2 $\overline{11} 0]$ orientation. More clearly resolved image contrast near the edge of the Au nanoparticle is found in the reconstructed phase compared to the $\mathrm{ABF}$ image. For the case of $\mathrm{GaN}$, the $\mathrm{Ga}-\mathrm{N}$ column pairs are clearly resolved in the reconstructed phase, but the contrast of nitrogen is next to invisible in the ABF image. The probe forming aperture for both datasets is about $14.4 \mathrm{mrad}$, and the synthetic ABF has a collection angle of 7.2-14.4 mrad. Both the Au nanoparticle and $\mathrm{GaN}$ datasets contain 128x128 probe positions, and were recorded with a camera speed of 1000 and $4000 \mathrm{fps}$, respectively. 